\title{
Web-Based Screening for Diabetic Retinopathy in a Primary Care Population: The EyeCheck Project
}

\author{
MICHAEL D. ABRAMOFF, M.D., Ph.D., ${ }^{1,2}$ and \\ MARIA S.A. SUTTORP-SCHULTEN, M.D., Ph.D. ${ }^{3}$
}

\begin{abstract}
The objective of this study was to evaluate the feasibility of ATA category 2 Web-based screening for diabetic retinopathy in a primary care population in the Netherlands. A total of 1,676 patients in a primary care setting, with diabetes, without known diabetic retinopathy, and without previous screening by an ophthalmologist, were included between January 1 and December 31, 2003. Patients underwent a brief questionnaire and two field retinal photography. Photographs were independently read by two ophthalmologists. Outcome measures were gradability of the photographs, need for pharmacologic pupil dilation, assessment as suspect for presence of diabetic retinopathy, of neovascularization and of diabetic retinopathy, and agreement between graders.

Of the population studied, $11.3 \%$ of patients required pupil dilation, average transmission time of images was $73 \mathrm{sec}, 12.0 \%$ of patients had ungradable photographs, $10.4 \%$ of the patients with gradable photographs were assessed as "suspect for diabetic retinopathy," and $2.0 \%$ were assessed to need urgent referral. Red lesions were present in $3.5 \%$ and bright lesions were present in $1.6 \%$ of all gradable patients. Neovascularization of the disk was found in one patient. Type 1 patients had much higher rates of "suspect for diabetic retinopathy" $(34.5 \%)$ than type 2 patients $(9.4 \%)$. Interrater agreement $\kappa$ was 0.93 .

Web-based screening, using open source technology and uncompressed images, is feasible in a primary care setting, with a high rate of inter-rater agreement. Dilate-as-needed may be a sensible approach for retinal photography. The high incidence of "suspect for diabetic retinopathy" in type 1 diabetes patients illustrates that web-based diabetic retinopathy screening programs for these patients may detect diabetic retinopathy that would otherwise have gone undetected.
\end{abstract}

\section{INTRODUCTION}

D IABETIC RETINOPATHY is the most important cause of blindness in the working population in the United States and in the European Union. ${ }^{1}$ Through early diagnosis and timely treatment, vision loss and blindness can largely be prevented. ${ }^{2-5}$ For a variety of reasons, only $50 \%$ of patients with diabetes are screened according to the guidelines and protocols of their respective countries. ${ }^{6,7}$ Digital retinal color fundus photography has been shown to have sensitivity and specificity comparable to indirect ophthalmoscopy by an ophthalmologist. 8,9 Computer-assisted diagnosis of lesions in such photographs is on the horizon. ${ }^{10-12}$

In 2000, the EyeCheck project was started in the Netherlands to try and solve such problems. It offers online screening for diabetic retinopathy over the Internet, nationwide, to any

\footnotetext{
${ }^{1}$ Department of Ophthalmology and Visual Sciences, University of Iowa Hospital and Clinics, and ${ }^{2}$ Department of Veterans' Affairs Medical Center, Iowa City, Iowa.

${ }^{3}$ Department of Ophthalmology, Vrije Universiteit University Hospital, Amsterdam, The Netherlands.
} 
primary care physician in the Netherlands. It conforms to the American Telemedicine Association Recommendations Category 2 for Diabetic Retinopathy Telediagnosis Systems. ${ }^{13,14}$ The purpose of the present study was to examine the impact of Web-based screening for diabetic retinopathy on a large primary care population by determining demographic and clinical characteristics over a single year, 2003.

\section{MATERIALS AND METHODS}

The setting for this retrospective study was a primary care population in family care physicians' offices or family physician laboratories. A physician or nurse trained to be able to diagnose and treat side effects of pharmacologic dilation drops was required to be present.

The inclusion criteria included diagnosis of diabetes at least one month previously, with the patient being interviewed and photographed at a primary care physician center for the EyeCheck project, with data submitted between January 1 and December 31, 2003.

Exclusion criteria included known diabetic retinopathy, previous inferior quality retinal photographs, and previous visit to an ophthalmologist for screening.

All patients gave informed consent after explanation at the end-user's site. No personalized health information was accessible to the researchers, and they only had access to aggregate data for the purpose of this study. The study followed the tenets of the Declaration of Helsinki.

\section{Technology applied}

Web technology: The methodology of the EyeCheck project was based on an online Web server system. This system was built using the Java programming language, from open source components exclusively, including image processing, data security, and data storage. ${ }^{15}$ Data security was provided by state-of-the-art, 512bit Secure Socket Layer (SSL) version 3.0 technology integrated into Java 2. ${ }^{16,17}$ Patient data was transmitted and stored using an Extended Markup Language (XML)-Hospital Language 7 (HL7) based data dictionary, and images were transmitted and stored uncompressed in the Digital Imaging and Communications in Medicine (DICOM) ophthalmology supplement 81 standard, in an open source Standard Query Language (SQL) database. ${ }^{18}$ The system conforms to the American Telemedicine Association Recommendations Category 2 for Diabetic Retinopathy Telediagnosis Systems. ${ }^{13,19}$ The EyeCheck project was initiated in 2000.

The system is fully Web-based, with online tools for configuration. All patient data and images are stored on a need-to-know basis, so that only those individuals that are allowed to access specific items of a submission are able to do so. For example, a "grader" is not allowed access to date of birth, street, city, telephone, or insurance status information. For this study, only aggregate information could be accessed, and access to any personal health information was not possible. The terminology for EyeCheck includes the terms "end-user site," "grader," and "submission." An "end-user site" is the location where patients with diabetes are examined, a "grader" is the person who in the Netherlands is required to be an ophthalmologist, to assess whether or not a patient's photographs are suspect for diabetic retinopathy, and a "submission" is the combination of patient data and retinal images.

Screening protocol: The protocol includes a brief Web-based questionnaire, measurement of visual acuity, and four retinal photographs, one disk centered and one fovea centered, for each eye. Fundus cameras for the EyeCheck project are required to have at least $768 \times 512$ 32-bit color resolution, and cameras used for this study were the Topcon NW100 (Topcon Europe, Rotterdam, the Netherlands) and Canon CR5-45NM (Canon, Kanagawa, Japan) nonmydriatic color fundus cameras set to $45^{\circ}$ field of view. Pharmacologic pupil dilation (Tropicamide $0.5 \%$ solution) was left to the discretion of the end-user, that is, a test image is taken and if deemed of sufficient quality (see below), no dilating drops are administered. The answers to the questionnaire together with the retinal photographs are then transmitted over the Internet and stored as a "submission." 
Table $1 . \quad$ Descriptive Statistics

\begin{tabular}{lcc}
\hline & Mean & 95\% Confidence interval \\
\hline Age (years) & 60.4 & $59.8-61.0$ \\
Gender (male) & $44.9 \%$ & $42.5-47.3 \%$ \\
Type 1 & $3.1 \%$ & $2.0-4.2 \%$ \\
Type 2 & $96.9 \%$ & $95.8-98.0 \%$ \\
HbA1C & $7.0 \%$ & $6.7-7.3 \%$ \\
Transmission speed & $73 \mathrm{sec}$ & $42-268 \mathrm{sec}$ \\
$\quad$ (all data) & & \\
\hline
\end{tabular}

Image quality

Submissions could be classified as nongradable by either end-user or expert reader (see below). Gradability criteria were available in a manual and were focus of the large vessels, visibility of at least one arteriole or capillary in both the upper and the lower half of the image, evenness of illumination, and absence of artifacts. If at least one image was nongradable, the submission was nongradable.

\section{Reading of retinal images}

The retinal photographs are graded using a browser and image processing tools available on the website. The result of the assessment can be either "not suspect," "suspect, not urgent" if a submission (at least one of the four images) is assessed to be suspect for diabetic retinopathy, "suspect, urgent" if a submission is assessed to require urgent referral to an ophthalmologist, or "not gradable," if one or more of the four images is below standard image quality as set forward in the protocol (see above). Additionally, if graders were certain that red lesions, namely, hemorrhages or microaneurysms; bright lesions, namely, exudates or retinal infarcts ("cotton wool spots"); or disk neovascularizations were present, they indicated the presence of these lesions. Graders were masked to each others' grading. If they disagreed, final grading was performed by a third grader who did have access to the two previous grades. After agreement by two or three graders, the grading process was closed, and the result of the assessment became immediately available at the "end-user site" on the Internet, where it can be stored or printed as a letter for the patient and the referring physician. If any suspicion of diabetic retinopathy was found, the patient was required to be referred to an ophthalmologist. Two retinal specialists (MDA, MSA) and one general ophthalmologist were graders in the EyeCheck project for the duration of this study.

Study parameters: Mean and 95\% confidence interval $[\mathrm{CI}]$ for age, time for data transmission to Web-server in seconds, serum $\mathrm{HbA} 1 \mathrm{C}$; and proportion and $95 \%$ proportion confidence interval for: gender, presence or absence of pupil dilation, type 1 or type 2 diabetes. Outcome measures were: proportion and 95\% proportion CI of presence of bad quality photographs, white lesions, red lesions, disk neovascularization (which because of its urgent nature is graded separately), retinal nevus, for each grader, "suspect, not urgent" and "suspect, urgent" assessment, and interrater agreement kappa $(\kappa)$ of this assessment. Ethnicity or race is not allowed to be recorded in the Netherlands.

\section{Statistics}

Mean, two rater agreement $\kappa$ and 95\% CIs were determined straightforwardly. Confidence intervals for proportions $p$ of dichotomous variables were estimated using the continuity correction:

$$
p=p \pm z\left(\sqrt{\frac{p(1-p)}{N}}+\frac{1}{2 N}\right)
$$

\section{RESULTS}

A total of 1,676 patients were included. Demographic data were consistent with an elderly type 2 diabetes population, see Table 1 . Only

Table 2. Diagnostic Results

\begin{tabular}{llcl}
\hline Images unreadable & $12 \%$ & $11 \%-14 \%$ & $\%$ of all \\
Not suspect for diabetic retinopathy & $89.6 \%$ & $89.3 \%-89.9 \%$ & $\%$ of gradable \\
Suspect for diabetic retinopathy & $10.4 \%$ & $10.2 \%-10.7 \%$ & $\%$ of gradable \\
\hline
\end{tabular}


Table 3. Specific Criteria for Diabetic Retinopathy

\begin{tabular}{llll}
\hline$\geq 1$ Red lesion present & $3.5 \%$ & $3.2-3.8 \%$ & $\%$ of gradable \\
$\geq 1$ Bright lesion present & $1.6 \%$ & $1.3-1.9 \%$ & $\%$ of gradable \\
Neovascularization of optic disk present & $0.07 \%$ & & $\%$ of gradable \\
Nevus present & $0.6 \%$ & & of gradable \\
\hline
\end{tabular}

$3.1 \%$ of patients $(95 \% \mathrm{CI}, 2 \%-4.2 \%)$ had type 1 diabetes. Average age was 60.4 years $(95 \% \mathrm{CI}$, 59.8 - 61.0 years), and $44.9 \%$ (95\% CI, $42.5 \%-$ $47.3 \%$ ) were male. Metabolic control was comparatively good as shown by an average HbA1C of $7.0 \%$ (95\% CI, 6.7\%-7.3\%). Pharmacologic pupil dilation was used in $11.3 \%$ (95\% CI, $9.3 \%-13.3 \%$ ) of patients. Transmission time of a submission was 73 seconds (95\% CI, 42 s-268 s).

Diagnostic results are summarized in Table 2. The retinal images were found not to be gradable in $12.0 \%$ of patients ( $95 \% \mathrm{CI}, 10.9 \%-13.1 \%$ ), and these patients were re-screened, and excluded from further study. Reasons for nongradability included all reasons in the protocol (see Materials and Methods section). Patients with nongradable photographs were somewhat older (65.1 years, 95\% CI, 63.4-66.8 years) than patients with gradable photographs (60.1 years, 95\% CI, 58.6-62.4 years). There was no difference in gender between these two groups.

Of all patients with gradable photographs, $10.4 \%$ (95\% CI, $8.9 \%-11.8 \%$ ) were assessed to be "suspect for diabetic retinopathy," while all other patients were found to be not suspect. $2.0 \%$ of all gradable patients $(95 \% \mathrm{CI}$, $1.3 \%-2.8 \%$ ) were assessed to need urgent referral. Patients assessed as "suspect" were slightly older (65.3 years, 95\% CI, 63.9-66.7) than patients not assessed as suspect (60.1 years, 95\% CI, 58.6-61.7). The HbA1C of patients assessed as "suspect" had a nonsignificant trend for higher $\mathrm{HbA} 1 \mathrm{C}$ (suspect 7.38\%, $95 \%$ CI, 6.84-7.91, not suspect $6.94 \%, 95 \%$ CI, 6.64-7.25.

An analysis of different lesions is shown in Table 3. Red lesions, including microaneurysms and hemorrhages, were present in 3.5\% (95\% CI, $3.4 \%-3.5 \%$ ) of all gradable patients, while bright lesions, including exudates and cottonwool spots, were present in $1.6 \%$ (95\% CI, $1.5 \%-1.6 \%$ ) of all gradable patients (Fig. 1A). Only $52 \%$ of all patients assessed as "suspect for diabetic retinopathy" had confirmed white or red lesions. Because neovascularization of the disk is an indicator of advanced diabetic retinopathy and need for immediate evaluation by an ophthalmologist, these were analyzed

B

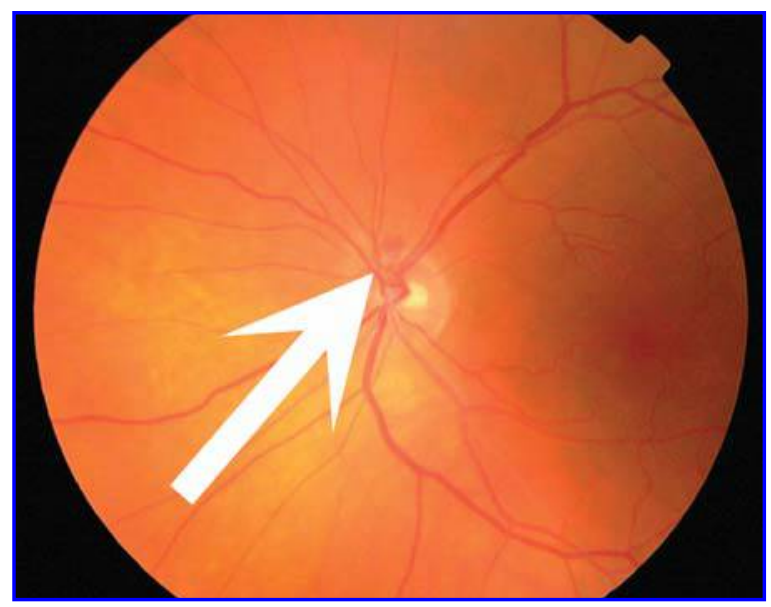

A

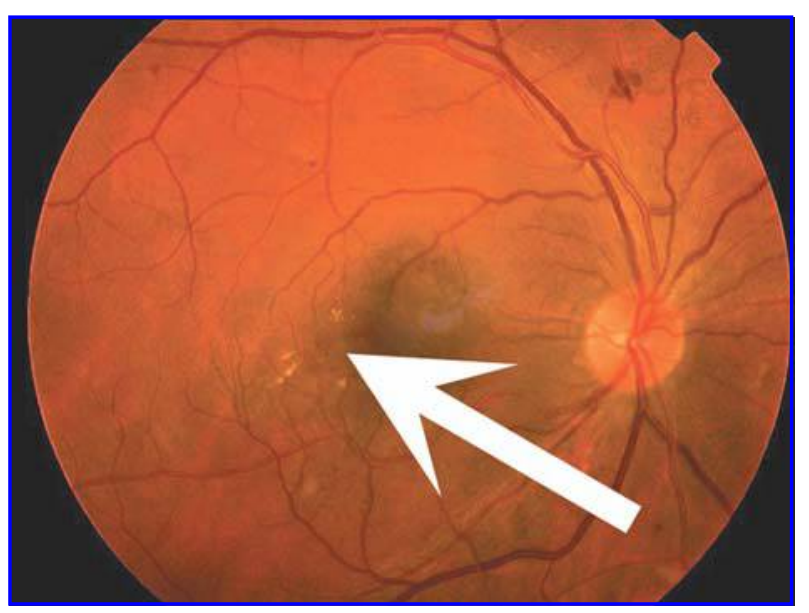


Table 4. Subanalysis of Patients Suspected of Diabetic Retinopathy

\begin{tabular}{lccc}
\hline Type 1, suspect & $34.5 \%$ & $16 \%-53 \%$ & $\%$ of all suspect \\
Type 2, suspect & $9.4 \%$ & $7.5 \%-11.4 \%$ & $\%$ of all suspect \\
Age, suspect & 60.1 & $58.6-61.7$ & years, of all suspect \\
Gender, suspect & $45.0 \%$ & $39.2-50.9 \%$ & $\%$ male, of all suspect \\
\hline
\end{tabular}

separately, and was found in one gradable patient $(0.7 \%$, Fig. 1$)$.

There was no difference in age or gender between patients suspect and nonsuspect for diabetic retinopathy, but type 1 patients were suspect much more often $(34.5 \%, 95 \% \mathrm{CI}$, $16 \%-53 \%)$ than type 2 patients $(9.4 \%, 95 \% \mathrm{CI}$, $7.5 \%-11.4 \%$, Table 4). An assessment not related to diabetic retinopathy, retinal nevus, was found in $6 \quad(95 \% \mathrm{CI}, 6 \%-6 \%)$ of gradable patients.

The agreement between graders measured as two observer $\kappa=0.93$ (85\% CI, 0.90-0.96).

\section{DISCUSSION}

Our results show that online screening for diabetic retinopathy is feasible, and that low cost open source and open standard technology can be used for this. We have shown that data transmission rates are sufficient for the needs of such projects. We found that less than $20 \%$ of patients in this population with diabetes, but without known diabetic retinopathy, required pharmacologic pupil dilation. This means that dilate-as-needed may be a reasonable approach in similar populations, balancing the need for adequate quality photographs versus the cardiovascular risk and the risk of angle closure glaucoma.

We found that the combination of camera resolution, number of photographs, and Webbased grading user interface ensures a high rate of intergrader reliability as evidenced by a $\kappa>$ 0.93. This high agreement seems to warrant that grading by two ophthalmologists can be done on an as-needed basis, that is, photographs are only graded by a second ophthalmologist if the grading ophthalmologist thinks that this is necessary.

Our study showed that in this relatively wellregulated population, the proportion of patients assessed as "suspect for diabetic reti- nopathy," and referred to an ophthalmologist, was approximately $10 \%$, and that the proportion of submissions with insufficient quality was approximately $12 \%$. The decision of whether or not to dilate pharmacologically was at the discretion of the end-user (the operator of the camera), and the reason was not recorded. Anecdotal evidence indicates that the usual reason for pharmacologic dilation was either inability to visualize the fundus or too dark fundus photographs. In almost half $(48 \%)$ of all patients that were assessed as "suspect for diabetic retinopathy," graders were not certain of the presence of red or bright lesions pathognomic for diabetic retinopathy. Patients "suspect for diabetic retinopathy" were older and had a nonsignificant tendency for higher $\mathrm{HbA} 1 \mathrm{C}$ in this study, confirming findings in earlier, less well-controlled, populations. ${ }^{20}$

The results may imply that the workload of ophthalmologists in such populations can be decreased by close to $75 \%$. The results also seem to indicate that the proportion of patients assessed as 'suspect for diabetic retinopathy', may be substantially higher than the number of patients with a true diagnosis of diabetic retinopathy. Just over half of the 'suspect' patients showed any evidence of hemorraghes, microaneurysms, cotton-wool spots, exudates, or neovascularizations. Consequently, the graders may have erred on the safe side and referred a considerable number of patients not because of overt diabetic retinopathy, but because of small retinal irregularities or 'artifacts' where the artifactual nature of a 'lesion' could not be confirmed. In this study, neovascularizations of the disk, a criterion for urgent referral to an ophthalmologist, was rare, occurring in only $0.7 \%$ of gradable patients. ${ }^{21}$

The results shows a much higher incidence of assessment as "suspect for diabetic retinopathy" in type 1 diabetes patients $(34.5 \%)$ compared to type 2 diabetes patients $(9.4 \%)$ in this 
population. This may be related to the fact that only patients without a previous visit to an ophthalmologist were included. A likely explanation is that most patients with type 1 diabetes, who are usually more aware of the importance of screening than type 2 patients, are already undergoing screening. The remaining patients, who for a variety of reasons had not been previously seen by an ophthalmologist, apparently have a much higher likelihood of diabetic retinopathy. This finding together with the low prevalence $(3.1 \%)$ of type 1 diabetes in this population, clearly illustrates the attractiveness of Web-based screening for this group of patients.

Other studies of Web-based screening have been published. ${ }^{421-22}$ However, to our knowledge, this is the first large-scale study, using published, open source standardized technology and uncompressed images, on feasibility, the impact of data transmission speed, proportion of nongradable photographs, frequency of pharmacologic pupil dilation, risk factors, and agreement between graders.

A drawback of this study is that it is a retrospective study, and that no independent confirmation of the accuracy, sensitivity, or specificity of the assessments is available. In other words, even though two ophthalmologists had to agree whether the photographs were suspect for diabetic retinopathy, and their inter-rater agreement was high, no ground truth from a reading center was obtained to evaluate their assessment. It cannot be determined from this study whether any diabetic retinopathy present in the photographs was missed by the graders or was present outside the field of view of the two field photographs. Other studies have shown that this risk is relatively small. ${ }^{8}$ Additionally, there are indications, as explained above, that the ophthalmologists were overly sensitive.

In summary, this study has shown that largescale screening for diabetic retinopathy over the Internet is feasible, that the technology can be based on open source technology and open standards, and that it allows a high rate of agreement between ophthalmologists. We are currently studying the relative risk for different risk factors, the effectiveness of computer-aided diagnosis, and the follow-up man- agement by referral ophthalmologists of the patients assessed as suspect, both in Iowa and the Netherlands. ${ }^{11,12,23}$ If employed on a larger scale, projects such as EyeCheck, especially if coupled with computer-aided diagnosis, can become a relatively low cost tool to reduce visual loss and blindness in patients with diabetes.

\section{ACKNOWLEDGMENTS}

Michael D. Abramoff is supported by a K-12 Career Development Award from the University of Iowa College of Medicine, Iowa; an unrestricted grant from Research to Prevent Blindness; the Wellmark Foundation; and by the Netherlands Organization for Health Research.

\section{REFERENCES}

1. Klonoff DC, Schwartz DM. An economic analysis of interventions for diabetes. Diabetes Care 2000; 23 : 390-404.

2. Chia DS, Yap EY. Comparison of the effectiveness of detecting diabetic eye disease: diabetic retinal photography versus ophthalmic consultation. Singapore Med I 2004; 45:276-279.

3. Early Treatment Diabetic Retinopathy Study Research Group. Early Photocoagulation for Diabetic Retinopathy: ETDRS report 9. Ophthalmology 1991; 98:766-785.

4. Aiello LM, Bursell SE, Cavallerano J, Gardner WK, Strong J. Joslin Vision Network Validation Study: pilot image stabilization phase. I Am Optom Assoc 1998; 69:699-710.

5. Bresnick GH, Mukamel DB, Dickinson JC, Cole DR. A screening approach to the surveillance of patients with diabetes for the presence of vision-threatening retinopathy. Ophthalmology 2000; 107:19-24.

6. Wilson C, Horton M, Cavallerano J, Aiello LM. The use of retinal imaging technology increases the rates of screening and treatment of diabetic retinopathy. Diabetes Care 2005; 28(2):318-322.

7. Brechner RJ, Cowie CC, Howie LJ, Herman WH, Will JC, Harris MI. Ophthalmic examination among adults with diagnosed diabetes mellitus. IAMA 1993; 270: 1714-1718.

8. Williams GA, Scott IU, Haller JA, Maguire AM, Marcus D, McDonald HR. Single-field fundus photography for diabetic retinopathy screening: a report by the American Academy of Ophthalmology. Ophthalmology 2004; 111:1055-1062.

9. van Leiden HA, Moll AC, Dekker JM, Abràmoff MD, Polak BCP. Photography or ophthalmoscopy for de- 
tection of diabetic retinopathy. Diabetes Care 2003; 26:1318-1319.

10. Niemeijer M, van Ginneken B, Staal JS, Suttorp-Schulten MS, Abràmoff MD. Automatic detection of red lesions in digital color fundus photographs. IEEE Trans Med Imag. 2005; 5(24):584-592.

11. Staal JS, Abramoff MD, Niemeijer M, Viergever MA, van Ginneken B. Ridge based vessel segmentation in color images of the retina. IEEE Trans Med Imaging 2004; 23:501-509.

12. Niemeijer M, Staal JS, van Ginneken B, Loog $M, A$ bràmoff MD. Comparative study of retinal vessel segmentation on a new publicly available database. Proc SPIE 2004;5370-5379.

13. University Health Network TC. HL7 Application Programming Interface. 2004. 10-1-2004.

14. American Telemedicine Association. Standards and Guidelines in Telehealth for Diabetic Retinopathy: American Telemedicine Association Position Statement, 2004.

15. Abràmoff MD, Magalhães PJ, Ram S. Image processing with ImageJ. Biophotonics 2004; 11:36-42.

16. Anderson RJ. Security in clinical information systems. London: British Medical Association, 1996.

17. Sun Microsystems I. Java 2 Secure Socket Extension Reference Guide. 2004.

18. Widenius M, Axmark D. MySQL Reference Manual . 1 ed. Sebastopol, CA: O'Reilly, 2004.

19. American Telemedicine Association OTSIG. Telehealth Practice Recommendations for Diabetic Retinopathy. 2004.
20. Vijan S, Hofer TP, Hayward RA. Cost-utility analysis of screening intervals for diabetic retinopathy in patients with type 2 diabetes mellitus. IAMA 2000; 283:889-896.

21. Gomez-Ulla F, Fernandez MI, Gonzalez F, Rey P, Rodriguez M, Rodriguez-Cid MJ, Casanueva FF, Tome MA, Garcia-Tobio J, Gude F. Digital retinal images and teleophthalmology for detecting and grading diabetic retinopathy. Diabetes Care 2002; 25:1384-1389.

22. Cavallerano AA, Cavallerano JD, Katalinic P, Tolson AM, Aiello LP, Aiello LM. Use of Joslin Vision Network digital-video nonmydriatic retinal imaging to assess diabetic retinopathy in a clinical program. Retina 2003; 23:215-223.

23. Niemeijer M, Ginniken Bv, Abràmoff MD. Automatic detection and classification of microaneurysms and small hemorrhages in color fundus photographs. Proc Computer Aided Fundus Imag Anal (CAFIA) 2003.

Address reprint requests to: Michael D. Abramoff, M.D., Ph.D. Department of Ophthalmology and Visual Sciences

University of Iowa Hospital and Clinics 200 Hawkins Drive Iowa City, IA 52242

E-mail: michael-abramoff@uiowa.edu 


\section{This article has been cited by:}

1. Jan Paulus, Jörg Meier, Rüdiger Bock, Joachim Hornegger, Georg Michelson. 2010. Automated quality assessment of retinal fundus photos. International Journal of Computer Assisted Radiology and Surgery . [CrossRef]

2. Michael D Abramoff, Meindert Niemeijer, Stephen R Russell. 2010. Automated detection of diabetic retinopathy: barriers to translation into clinical practice. Expert Review of Medical Devices 7:2, 287-296. [CrossRef]

3. Meindert Niemeijer, Bram van Ginneken, Michael J. Cree, Atsushi Mizutani, GwÉnolÉ Quellec, Clara I. Sanchez, Bob Zhang, Roberto Hornero, Mathieu Lamard, Chisako Muramatsu, Xiangqian Wu, Guy Cazuguel, Jane You, AgustÍn Mayo, Qin Li, Yuji Hatanaka, BÉatrice Cochener, Christian Roux, Fakhri Karray, MarÍa Garcia, Hiroshi Fujita, Michael D. Abramoff. 2010. Retinopathy Online Challenge: Automatic Detection of Microaneurysms in Digital Color Fundus Photographs. IEEE Transactions on Medical Imaging 29:1, 185-195. [CrossRef]

4. Herman Bartling, Peter Wanger, Lene Martin. 2009. Automated quality evaluation of digital fundus photographs. Acta Ophthalmologica 87:6, 643-647. [CrossRef]

5. Jochanan Benbassat, Bettine C. P. Polak. 2009. Reliability of screening methods for diabetic retinopathy. Diabetic Medicine 26:8, 783-790. [CrossRef]

6. Meindert Niemeijer, Michael D. Abramoff, Bram van Ginneken. 2009. Information Fusion for Diabetic Retinopathy CAD in Digital Color Fundus Photographs. IEEE Transactions on Medical Imaging 28:5, 775-785. [CrossRef] 